\title{
Chronotype Is Associated with Emotional Dysregulation Influenced by Childhood Trauma: A Retrospective Study
}

\author{
Young-Min Park \\ Department of Psychiatry, Ilsan Paik Hospital, Inje University College of Medicine, Goyang, Korea
}

\begin{abstract}
Objective: The aim of this study was to clarify the relationships among childhood trauma, emotional dysregulation, and circadian preference in patients with major depressive disorder (MDD). Methods: This study included 107 outpatients aged between 20 and 65 years who met the criteria of the fifth version of the Diagnostic and Statistical Manual of Mental Disorders for MDD on their medical records. The subjects were divided into morningness, intermediate, and eveningness groups according to their chronotype based on the Korean version of the Composite Scale of Morningness (K-CSM). All of the patients were evaluated using the Korean version of the Mood Disorder Questionnaire (K-MDQ) and the Beck Depression Inventory (BDI). Childhood traumatic events were evaluated using the Korean version of the Childhood Trauma Questionnaire (K-CTQ). Results: Comparing the demographic and clinical variables revealed that the mean age and the emotional abuse (EA), total BDI, and total K-MDQ scores differed significantly among the three groups. The total K-CSM score was negatively correlated with the total KMDQ, K-CTQ, EA, and emotional neglect scores. The K-MDQ score was positively correlated with the total K-CTQ, EA, and PA scores. Linear regression analyses were performed to test whether childhood trauma was driving the association with emotional dysregulation (using $\mathrm{K}$ $\mathrm{MDQ}$ ), and whether emotional dysregulation was driving the association with eveningness (using K-CSM). Both higher EA and total BDI scores were independently associated with a higher K-MDQ score. A higher K-MDQ score was also independently associated with a lower KCSM score (toward eveningness). Conclusion: The findings of this study support that childhood trauma influences emotional dysregulation, which in turn leads to a circadian preference toward eveningness. It can therefore be assumed that emotional dysregulation mediates the relationship between childhood trauma and eveningness.
\end{abstract}

Key Words: Childhood trauma; Emotional dysregulation; Chronotype; Major depressive disorder

Received: December 26, 2018 Accepted: January 12, 2019

Corresponding author: Young-Min Park, MD, PhD, Department of Psychiatry, Ilsan Paik Hospital, Inje University College of Medicine, 170 Juhwa-ro, Ilsanseo-gu, Goyang 10380, Korea.

Tel: 82-31-910-7260, Fax: 82-31-910-7268, E-mail: medipark@hanmail.net

(a) This is an Open Access article distributed under the terms of the Creative Commons Attribution Non-Commercial License (https://creativecommons.org/licenses/bync/4.0) which permits unrestricted non-commercial use, distribution, and reproduction in any medium, provided the original work is properly cited.

\section{INTRODUCTION}

Mood disorder causes abnormalities in biological rhythms [1,2]. In particular, patients with mood disorder exhibit differences in chronotype from the normal population, such as in the circadian preference [3]. A study of the general population found that people with depressive symptoms were more likely to exhibit eveningness than those without depressive symptoms [4]. A study of patients with major depressive disorder (MDD) also found that the frequency of eveningness was higher than that in normal controls [5].

However, not all patients with MDD exhibit eveningness, with both morningness and an intermediate type also being found. This makes it likely that other factors influence the circadian preference of patients with MDD.

This study tested the hypothesis that childhood trauma such as abuse or neglect causes emotional dysregulation and that it acts as a mediator and affects the chronotype. Emotional dysregulation was measured using the Korean version of the Mood Disorder Questionnaire (K-MDQ), whose items seem to reflect not only the experience characteristic of bipolar disorder (BD) but also the emotional dysregulation and risky behaviors that are characteristic of borderline personality disorder [6]. Therefore, the aim of this study was to clarify the relationships among 
Table 1. Comparison of demographic and clinical variables between morningness, intermediate, and eveningness patients with major depressive disorder

\begin{tabular}{lcccc}
\multicolumn{1}{c}{ Variable } & Morningness $(\mathrm{n}=13)$ & Intermediate $(\mathrm{n}=61)$ & Eveningness $(\mathrm{n}=32)$ & $\mathrm{p}$ \\
Age, years & $36.54 \pm 11.54$ & $34.60 \pm 10.02$ & $29.62 \pm 11.48$ & $0.029^{\dagger}$ \\
Sex, male/female* & $7 / 6$ & $19 / 42$ & $11 / 21$ & 0.30 \\
Total K-CTQ score & $40.12 \pm 13.25$ & $51.07 \pm 18.34$ & $50.97 \pm 18.62$ & 0.084 \\
$\quad$ Emotional abuse score & $6.54 \pm 2.26$ & $9.79 \pm 5.20$ & $10.94 \pm 4.77$ & $0.017^{\dagger}$ \\
Physical neglect score & $9.88 \pm 4.83$ & $10.21 \pm 3.95$ & $9.34 \pm 3.71$ & 0.43 \\
Physical abuse score & $7.38 \pm 2.96$ & $10.16 \pm 5.42$ & $9.66 \pm 5.60$ & 0.19 \\
Sexual abuse score & $5.85 \pm 1.41$ & $6.93 \pm 3.88$ & $6.78 \pm 3.84$ & 0.88 \\
$\quad$ Emotional neglect score & $10.46 \pm 4.59$ & $13.97 \pm 6.32$ & $14.25 \pm 5.88$ & 0.086 \\
Total BDI score & $21.38 \pm 12.18$ & $23.11 \pm 11.21$ & $29.77 \pm 8.97$ & $0.0069^{\ddagger}$ \\
Total K-MDQ score & $4.23 \pm 2.09$ & $5.08 \pm 3.31$ & $7.31 \pm 3.40$ & $20.97 \pm 4.08$ \\
Total K-CSM score & $43.35 \pm 2.25$ & $32.52 \pm 3.71$ & $0.0034^{\ddagger}$ & $0.00^{\ddagger}$ \\
\hline
\end{tabular}

Data are mean \pm SD values. Comparisons were made using the Kruskal-Wallis test (*chi-square test). ${ }^{\dagger} \mathrm{p}<0.05$, ${ }^{\ddagger} \mathrm{p}<0.01$. K-CTQ: Korean version of the Childhood Trauma Questionnaire, BDI: Beck Depression Inventory, K-MDQ: Korean version of the Mood Disorder Questionnaire, K-CSM: Korean version of the Composite Scale of Morningness

childhood trauma, emotional dysregulation, and chronotype in patients with MDD.

\section{METHODS}

This study included 107 outpatients aged between 20 and 65 years who met the criteria of the fifth version of the Diagnostic and Statistical Manual of Mental Disorders (DSM-5) for MDD at Ilsan Paik Hospital between 2014 and 2018 based on their medical records. Subjects who had psychotic symptoms, any additional mental disorders, or personality disorders according to DSM-5 were excluded. None of the subjects had a history of hypomanic or manic episodes.

The subjects were divided into morningness, intermediate, and eveningness groups according to their chronotype based on the Korean version of the Composite Scale of Morningness (K-CSM), which was derived from the widely used Horne-Ostberg scale [7]. Several studies have demonstrated the good test-retest reliability and adequate external validity of the Composite Scale of Morningness $[8,9]$.

The Korean version of the Childhood Trauma Questionnaire (K-CTQ) is a self-report questionnaire comprising the following five subscales related to the experience of childhood abuse or neglect: physical abuse (PA), emotional abuse (EA), sexual abuse (SA), physical neglect (PN), and emotional neglect (EN). Each subscale consists of five items that are rated on a 5-point scale, from 1 (never true) to 5 (very often true). Depression was evaluated using the Beck Depression Inventory (BDI) and the Korean version of the Mood Disorder Questionnaire (K-MDQ). The KMDQ was used to identify subjects who were positive for bipolarity or emotional dysregulation.

Analysis of variance or the Kruskal-Wallis test, chi-square test, and Pearson's or Spearman's correlation test were used to perform group comparisons according to the properties of variables. Multiple linear regression was used to identify the associations be-

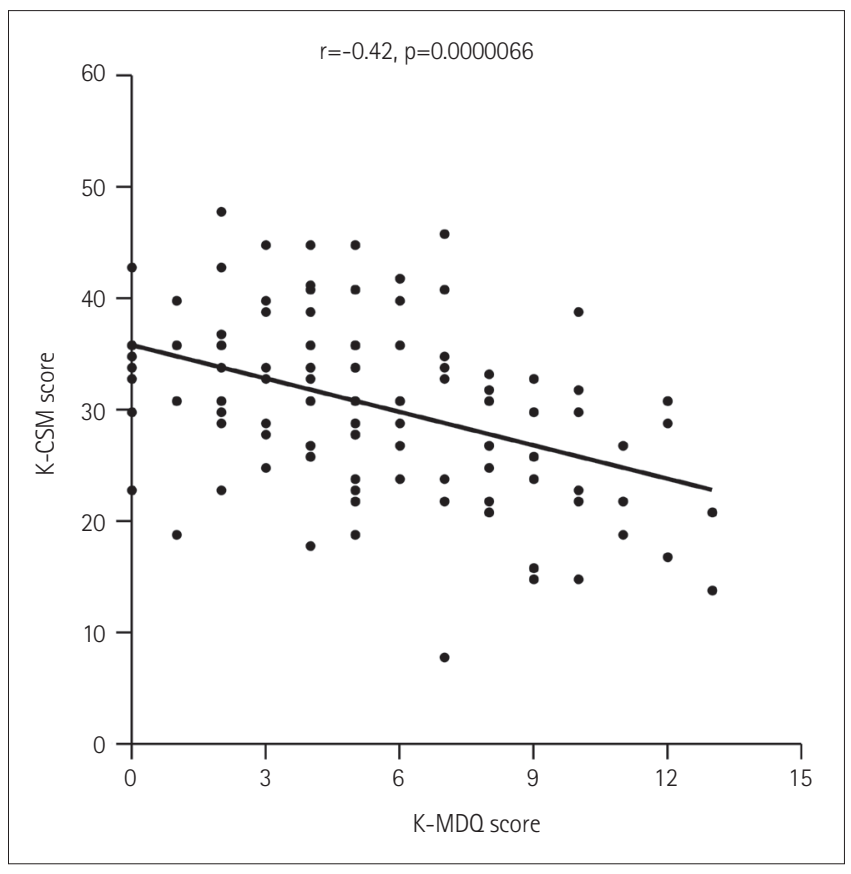

Figure 1. The correlation between the total K-MDQ and K-CSM score. K-MDQ: Korean version of the Mood Disorder Questionnaire, K-CSM: Korean version of the Composite Scale of Morningness.

tween K-CSM, K-MDQ, and K-CTQ scores.

The study protocol was approved by the ethics committee of Ilsan Paik Hospital before beginning the investigation (ISPAIK 2018-10-015-001).

\section{RESULTS}

The demographic and clinical variables in the morningness, intermediate, and eveningness groups are compared in Table 1. The mean age and the EA, total BDI, and total K-MDQ scores differed significantly among the three groups, whereas there were no significant intergroup differences in the total K-CTQ, PA, SA, EN, 
or PN score.

The number of subjects with experience of childhood trauma differed significantly among the three groups $\left(\chi^{2}=6.1, \mathrm{df}=2, \mathrm{p}=\right.$ $0.047)$. In addition, the total K-CSM score was negatively correlated with the total K-MDQ (Figure 1), K-CTQ, EA, and EN scores. The K-MDQ score was positively correlated with the total K-CTQ (Figure 2), EA (Figure 3), and PA (Figure 4) scores.

Linear regression analyses were performed to test whether

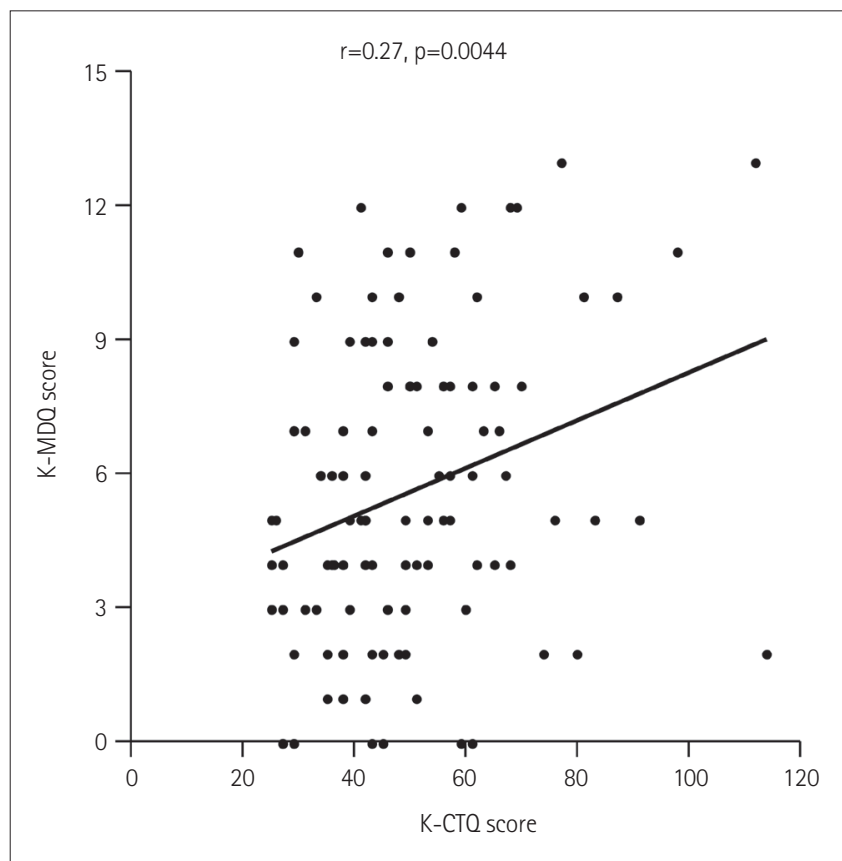

Figure 2. The correlation between the total K-CTQ and K-MDQ score. K-CTQ: Korean version of the Childhood Trauma Questionnaire, KMDQ: Korean version of the Mood Disorder Questionnaire.

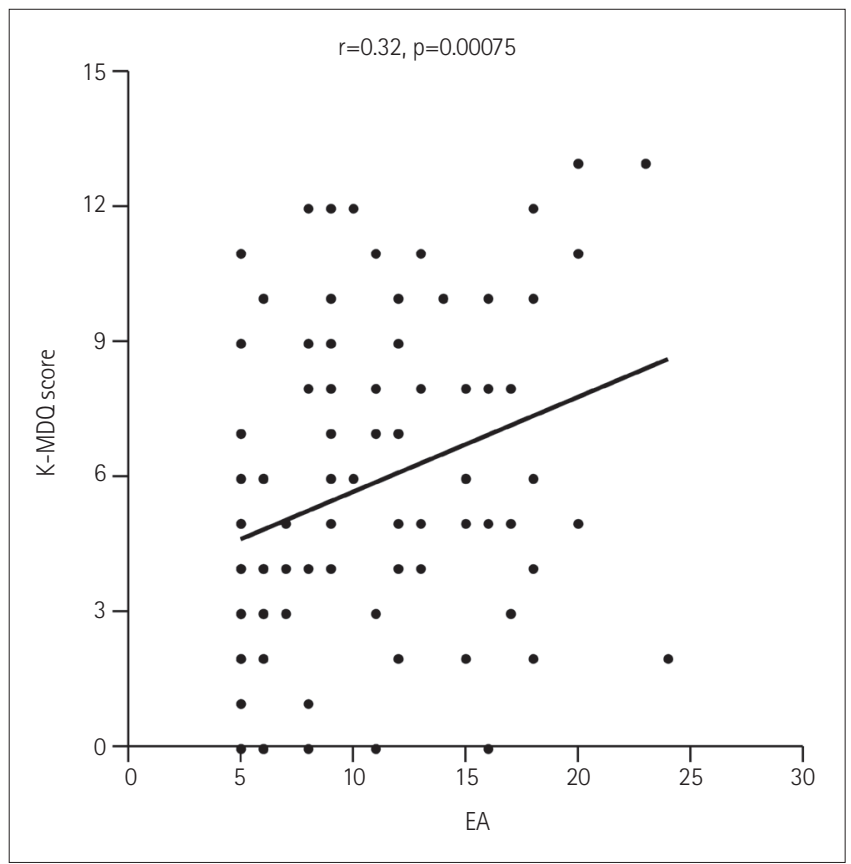

Figure 3. The correlation between the EA and total K-MDQ score. EA: emotional abuse, K-MDQ: Korean version of the Mood Disorder Questionnaire. childhood trauma (K-CTQ) was driving the association with emotional dysregulation (K-MDQ) and whether emotional dysregulation (K-MDQ) was driving the association with chronotype (K-CSM). Both higher EA and total BDI scores were independently associated with a higher K-MDQ score (Table 2). A higher K-MDQ score was also independently associated with a lower K-CSM score (toward eveningness) (Table 2). In contrast, the EA

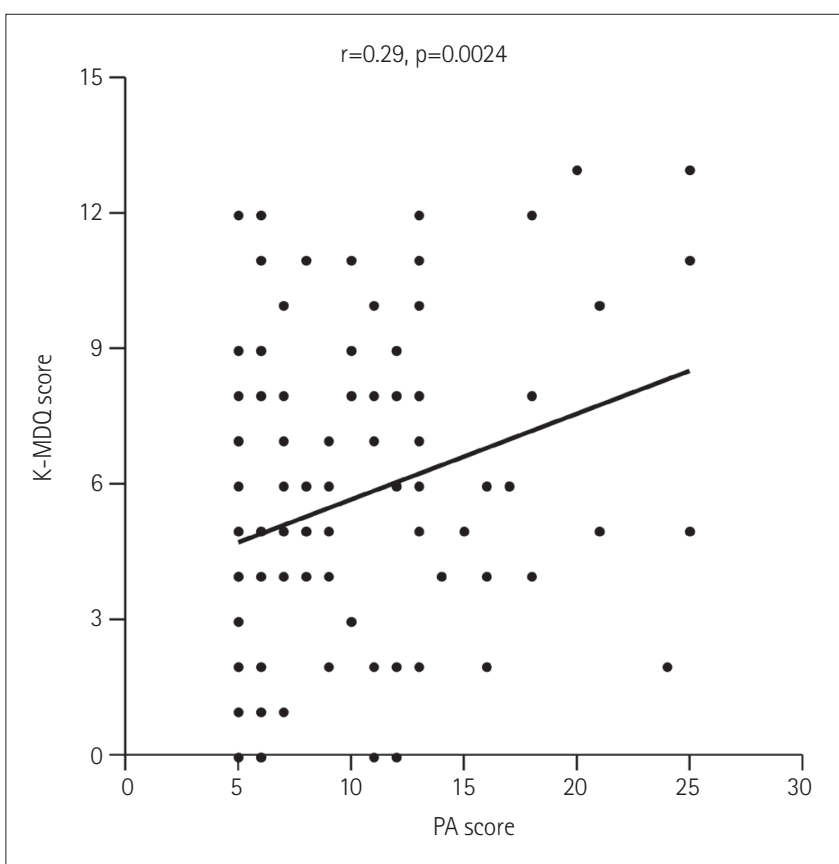

Figure 4. The correlation between the PA and total K-MDQ score. PA: physical abuse, K-MDQ: Korean version of the Mood Disorder Questionnaire.

Table 2. Results of multiple linear regression analyses of the associations between the K-MDQ score, childhood trauma parameters, and the K-CSM score

\begin{tabular}{lllll}
\multicolumn{1}{c}{ Variable } & Coefficient & \multicolumn{1}{c}{ SE } & \multicolumn{1}{c}{$\mathrm{t}$} & \multicolumn{1}{c}{$\mathrm{p}$} \\
Total K-MDQ score & & & & \\
$\quad$ Age & -0.012 & 0.029 & -0.42 & 0.67 \\
Sex & -0.42 & 0.68 & -0.059 & 0.54 \\
EA & 0.34 & 0.14 & 2.44 & $0.016^{*}$ \\
PA & 0.37 & 0.17 & 2.15 & $0.034^{*}$ \\
ExP abuse & -0.023 & 0.025 & -0.95 & 0.34 \\
Goodness of fit & & & & $\mathrm{R}^{2}=0.1$
\end{tabular}

Total K-CSM score

$\begin{array}{lclrl}\text { Age } & 0.12 & 0.066 & 1.83 & 0.071 \\ \text { Sex } & -0.45 & 1.60 & -0.28 & 0.78 \\ \text { EA } & 0.12 & 0.33 & 0.36 & 0.72 \\ \text { PA } & 0.43 & 0.39 & 1.11 & 0.27 \\ \text { E×P abuse } & -0.023 & 0.025 & -0.95 & 0.34 \\ \text { Total K-MDQ score } & -0.78 & 0.23 & -3.35 & 0.0012^{\dagger} \\ \text { Total BDI score } & -0.18 & 0.076 & -2.35 & 0.021^{*} \\ \text { Goodness of fit } & & & & \mathrm{R}^{2}=0.22\end{array}$

$\mathrm{E} \times \mathrm{P}$, interaction between emotional and physical abuse. ${ }^{*} \mathrm{p}<0.05,{ }^{\dagger} \mathrm{p}<$ 0.01. K-MDQ: Korean version of the Mood Disorder Questionnaire, K-CSM: Korean version of the Composite Scale of Morningness, EA: emotional abuse, PA: physical abuse, BDI: Beck Depression Inventory 
and PA scores were not associated with the K-CSM score, unlike in the correlation test.

\section{DISCUSSION}

This study found that childhood trauma influenced emotional dysregulation, which in turn also the chronotype. It can therefore be assumed that emotional dysregulation mediates the relationship between childhood trauma and chronotype. There have been some reports of $\mathrm{MDD}$ and $\mathrm{BD}$ being related to eveningness [10,11], but the present study is the first to find a relationship between stronger emotional dysregulation in patients with MDD and eveningness.

Many studies have found childhood trauma to have robust negative impacts on clinical outcomes such as earlier age at onset, more severe type, more mood episodes, psychotic episodes, suicide attempts, mixed symptoms or episodes, comorbidity of substance abuse or dependence, and worse life functioning in mood disorder [12-14]. Our results also indicate that childhood trauma aggravates the severity of depression, which is consistent with previous findings $[15,16]$. In addition, childhood trauma has been reported to cause emotional dysregulation $[15,17,18]$, as also found in the present study. However, there are no previous reports of either emotional dysregulation or childhood trauma influencing the chronotype.

A particularly interesting finding of this study was that higher scores for EA and PA strengthened the association with eveningness. However, there was no significant relationship between EA and PA scores and eveningness when age, sex, emotional dysregulation, and severity of depression were controlled. It can be assumed that EA and PA do not directly affect the chronotype, instead affecting it via emotional dysregulation.

The K-MDQ was originally designed as a screening test for $\mathrm{BD}$ [19]. However, the subjects in the current study did not have a history of any (hypo) manic episodes, and some studies have shown that individuals with emotional dysregulation (e.g., borderline personality disorder) can exhibit higher scores on the Mood Disorder Questionnaire [6,20].

In a large Internet-based surveillance study, cyclothymic and euphoric temperaments (which are related to BD) and apathetic, volatile, and disinhibited temperaments (which are related to attention-deficit/hyperactivity disorder) were associated with eveningness [21]. In addition, eveningness is associated with less emotional control, coping, volition, and caution, and more affective instability and externalization [21].

The findings of this study support that childhood trauma influences emotional dysregulation, which in turn leads to a circadian preference toward eveningness. It can therefore be assumed that emotional dysregulation mediates the relationship between childhood trauma and eveningness.

\section{Acknowledgments}

This article was supported by a grant from National Research Foundation of Korea (NRF), funded by Ministry of Education (NRF-2018 R1D1A1A02085847).
Conflicts of Interest

The author has no potential conflicts of interest to disclose.

\section{ORCID iD}

Young-Min Park (1)

https://orcid.org/0000-0002-4993-1426

\section{REFERENCES}

1. Melo MCA, Abreu RLC, Linhares Neto VB, de Bruin PFC, de Bruin VMS. Chronotype and circadian rhythm in bipolar disorder: a systematic review. Sleep Med Rev 2017;34:46-58.

2. Regestein QR, Monk TH. Delayed sleep phase syndrome: a review of its clinical aspects. Am J Psychiatry 1995;152:602-608.

3. Selvi Y, Aydin A, Boysan M, Atli A, Agargun MY, Besiroglu L. Associations between chronotype, sleep quality, suicidality, and depressive symptoms in patients with major depression and healthy controls. Chronobiol Int 2010; 27:1813-1828.

4. Merikanto I, Kronholm E, Peltonen M, Laatikainen T, Vartiainen E, Partonen T. Circadian preference links to depression in general adult population. J Affect Disord 2015;188:143-148.

5. Müller MJ, Kundermann B, Cabanel N. Eveningness and poor sleep quality independently contribute to self-reported depression severity in psychiatric inpatients with affective disorder. Nord J Psychiatry 2016;70:329-334.

6. Balling C, Chelminski I, Dalrymple K, Zimmerman M. Differentiating borderline personality from bipolar disorder with the Mood Disorder Questionnaire (MDQ): a replication and extension of the International Mood Network (IMN) Nosology Project. Compr Psychiatry 2019;88:49-51.

7. Drennan MD, Klauber MR, Kripke DF, Goyette LM. The effects of depression and age on the Horne-Ostberg morningness-eveningness score. J Affect Disord 1991;23:93-98.

8. Jankowski KS. Composite Scale of Morningness: psychometric properties, validity with Munich ChronoType Questionnaire and age/sex differences in Poland. Eur Psychiatry 2015;30:166-171.

9. Rahimi Pordanjani T, Ebrahimi AM. Psychometric properties of Persian version of Composite Scale of Morningness. Asian J Psychiatr 2017;25:260-266.

10. Kitamura S, Hida A, Watanabe M, Enomoto M, Aritake-Okada S, Moriguchi $\mathrm{Y}$, et al. Evening preference is related to the incidence of depressive states independent of sleep-wake conditions. Chronobiol Int 2010;27:1797-1812.

11. Ahn YM, Chang J, Joo YH, Kim SC, Lee KY, Kim YS. Chronotype distribution in bipolar I disorder and schizophrenia in a Korean sample. Bipolar Disord 2008;10:271-275.

12. Jaworska-Andryszewska P, Rybakowski JK. Childhood trauma in mood disorders: neurobiological mechanisms and implications for treatment. Pharmacol Rep 2018;71:112-120.

13. Daruy-Filho L, Brietzke E, Lafer B, Grassi-Oliveira R. Childhood maltreatment and clinical outcomes of bipolar disorder. Acta Psychiatr Scand 2011;124: 427-434.

14. Infurna MR, Reichl C, Parzer P, Schimmenti A, Bifulco A, Kaess M. Associations between depression and specific childhood experiences of abuse and neglect: a meta-analysis. J Affect Disord 2016;190:47-55.

15. Huh HJ, Kim KH, Lee HK, Chae JH. The relationship between childhood trauma and the severity of adulthood depression and anxiety symptoms in a clinical sample: the mediating role of cognitive emotion regulation strategies. J Affect Disord 2017;213:44-50.

16. Vares EA, Salum GA, Spanemberg L, Caldieraro MA, Souza LH, Borges Rde $\mathrm{P}$, et al. Childhood trauma and dimensions of depression: a specific association with the cognitive domain. Braz J Psychiatry 2015;38:127-134.

17. Dvir Y, Ford JD, Hill M, Frazier JA. Childhood maltreatment, emotional dysregulation, and psychiatric comorbidities. Harv Rev Psychiatry 2014;22:149161.

18. Hopfinger L, Berking M, Bockting CL, Ebert DD. Emotion regulation mediates the effect of childhood trauma on depression. J Affect Disord 2016;198: 189-197.

19. Miller CJ, Klugman J, Berv DA, Rosenquist KJ, Ghaemi SN. Sensitivity and specificity of the Mood Disorder Questionnaire for detecting bipolar disorder. J Affect Disord 2004;81:167-171. 
20. Vöhringer PA, Barroilhet SA, Alvear K, Medina S, Espinosa C, Alexandrovich K, et al. The International Mood Network (IMN) Nosology Project: differentiating borderline personality from bipolar illness. Acta Psychiatr Scand
2016;134:504-510

21. Ottoni GL, Antoniolli E, Lara DR. Circadian preference is associated with emotional and affective temperaments. Chronobiol Int 2012;29:786-793. 